\title{
Physical capability, physical activity, and their association with femoral bone mineral density in adults aged 40 years and older: The Tromsø study 2015-2016
}

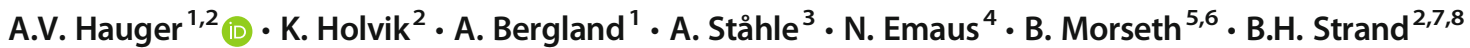

Received: 2 July 2020 / Accepted: 1 April 2021 / Published online: 16 April 2021

(C) The Author(s) 2021

\begin{abstract}
Summary Since muscles can influence bone growth and vice versa, we examined if level of physical activity and physical capability tests can predict areal bone mineral density (aBMD). Both high activity level and good test performance were associated with higher aBMD, especially in women.

Introduction Muscle influences bone formation and vice versa. Tests of physical capability and level of physical activity reflect various muscle qualities. We assessed the associations between total hip aBMD and physical activity as well as a range of standardized physical capability tests in an adult general population.

Methods A total of 3533 women and men aged 40-84 years, participating in the population-based cross-sectional Tromsø study in Norway in 2015-2016, were included. Linear regression was used to assess associations between aBMD and physical activity and the physical capability tests grip strength, Timed Up and Go (TUG), Short Physical Performance Battery (SPPB), and standing balance. Non-linear associations were examined in cubic spline models. Standardized regression coefficients were calculated to compare effect sizes across physical capability measures.

Results In fully adjusted models, higher physical activity was positively associated with total hip aBMD in both sexes compared to a sedentary lifestyle. All tests of physical capability were associated with aBMD in women, SPPB showing the strongest association although effect sizes were too small to indicate clinically significant differences (1 point increase corresponded to an aBMD increase of $0.009 \mathrm{~g} / \mathrm{cm}^{2}, \mathrm{CI}=0.005$ to 0.012 ). In men, $\mathrm{SPPB}$ and its subtests were associated with aBMD with chair rises showing the strongest association ( $1 \mathrm{~s}$ increase in execution time corresponded to an aBMD decrease of $0.005 \mathrm{~g} / \mathrm{cm}^{2}, \mathrm{CI}=0.008$ to 0.002 ).

Conclusion Physical activity was associated with aBMD, and tests of physical capability can account for some of the aBMD variations in adults aged 40 years and older, especially in women.
\end{abstract}

Keywords Bone mineral density $\cdot$ Osteoporosis $\cdot$ Physical activity $\cdot$ Physical capability $\cdot$ Physical function

A.V. Hauger

avha@fhi.no; annettevh@hotmail.com

1 Department of Physiotherapy, Faculty of Health Sciences, OsloMetOslo Metropolitan University, Postboks 4, St. Olavs plass, 0130 Oslo, Norway

2 Department of Chronic Diseases and Ageing, Norwegian Institute of Public Health, Marcus Thranes gate 6, 0473 Oslo, Norway

3 Department of Neurobiology, Care Sciences and Society, Division of Physiotherapy, Karolinska Institutet, Alfred Nobels Allé 23, 14183 Huddinge, Sweden
4 Department of Health and Care Sciences, Faculty of Health Sciences, UiT The Arctic University of Norway, 9037 Tromsø, Norway

5 Department of Community Medicine, University of Troms $\varnothing$, Tromsø, Norway

6 Department of Clinical Therapeutic Services, University Hospital of Northern Norway, Tromsø, Norway

7 Norwegian National Advisory Unit on Ageing and Health, Vestfold Hospital Trust, Tønsberg, Norway

8 Department of Geriatric Medicine, Oslo University Hospital, Oslo, Norway 


\section{Introduction}

Muscle tissue influences the formation of bone tissue and vice versa [1], and there is a positive association between muscle mass and bone mineral density (BMD) [2-5]. From midlife, BMD decreases continuously in both women and men but more rapidly in women during the first years following menopause [6]. Osteoporosis and sarcopenia, the latter defined by both loss of muscle mass and loss of physical capability [7], share many of the same risk factors such as old age, low socioeconomic status, poor nutrition, and inactivity, and the two conditions are often present in the same individuals $[7,8]$.

Several review articles have tried to quantify the effect of physical activity on measured BMD and to identify activities with the strongest effect on bone formation, both in general healthy populations and in people with osteoporosis [9-11]. Results indicate that weight-bearing exercise has the potential to be a safe and effective way to avert bone loss in both women and men, though the effects reported so far have been small to moderate [9-14].

Self-reported level of physical activity, although prone to reporting bias, is easily obtained and is reported to be associated with BMD $[15,16]$. Physical capability tests aim to assess individual capacity to undertake the physical tasks of daily living [17]. Lower scores on such tests, including measures of locomotion, muscle strength, and balance, are associated with greater risk of subsequent disability and morbidity. Consequently, favorable scores on such measures have been suggested as indicators of healthy aging [17]. BMD is strongly related to aging, and studies have revealed associations between physical capability indicators and BMD, but the strength of these associations seems to depend on the specific physical capability indicator [18-23]. Identification of factors that account for variations in BMD among adults during midlife and older age may guide intervention strategies for enhanced BMD in this group.

While walking speed does not seem to be a strong predictor of BMD, studies have consistently found a positive association between hand grip strength and BMD in the upper limbs $[18,19]$. An association between grip strength and lower limb BMD remains inconclusive $[18,20]$. We recently reported an inverse association between grip strength and fracture risk in the population-based Tromsø study, including hip fractures [24]. The ability to stand on one leg over time is a predictor of hip fracture risk $[25,26]$, and several studies have indicated that one-leg stance tests are associated with BMD [21-23]. Other physical capability tests, such as the Short Physical Performance Battery (SPPB) and Timed Up and Go (TUG), are often performed when assessing older people's physical capability, to measure the severity of sarcopenia [7] and to evaluate risk of falling [27-29]. However, few studies have investigated the association between these tests and BMD.
Since improving physical capability and level of physical activity is achievable for most people, it is important to investigate if these modifiable factors contribute to explain variations in BMD in an ambulatory population, as a basis for planning preventive measures.

We therefore aimed to investigate whether a range of physical capability tests (grip strength, TUG, SPPB, and standing balance) and self-reported level of physical activity were associated with areal BMD (aBMD) in the total hip, independently of age, comorbidities, and other health- and lifestylerelated variables in adults during midlife and older age in the population-based Tromsø study. We also aimed to compare the strength of the tests' associations with aBMD internally in the population.

We hypothesized that those with low levels of physical activity or lower performance on the physical capability tests had lower aBMD in the hip. To our knowledge, this is the first study to assess the relationship between aBMD and several commonly used tests of physical capability and self-reported physical activity in a large general population-based study where the strengths of the associations within the population are also compared.

\section{Methods}

\section{Study design, setting, and participants}

The present study used cross-sectional data from the Tromsø study in Norway. The Tromsø study was initiated in 1974 and is a population-based, longitudinal study focusing on lifestyle-related diseases and associated risk factors [30]. It has been repeated seven times, with 6 to 7 years between study waves. Our study was based on data from the 7th wave (Tromsø 7), which was conducted in 2015-2016. All inhabitants in Tromsø municipality aged 40 years and older, 16535 women and 16052 men in total, were eligible to participate and invited to Tromsø 7. Altogether, 11074 women and 10009 men participated. As part of the additional testing, a random sample of the participants was invited to areal BMD measurements. Of 5 232 invited, 3658 (70\%) participants aged 40-99 years (mean age 66.1, SD 9.0) had their total hip aBMD measured and were eligible for the present study. The final study population included participants with completed aBMD measurement at the total hip and available information about height, weight, smoking, education level, and self-reported chronic diseases $(n=3533)$ who completed at least one of the physical performance tests: grip strength (3 403), TUG (3 393), SPPB (3 409), or one-leg stance with eyes closed (3 159), or answered the question regarding level of leisure time physical activity (3 394). See Fig. 1 for flow chart of included participants. Mean 
age for the included participants was 65.8 (SD 9.0) ranging from 40 to 84 years. Descriptive statistics for the population are presented in Table 1.

\section{Outcome measures}

\section{aBMD}

Total hip aBMD expressed as $\mathrm{g} / \mathrm{cm}^{2}$ was measured by dualenergy X-ray absorptiometry (DXA) (GE Lunar Prodigy, LUNAR Corporation, Madison, WI, USA). The scans were performed by specially trained technicians according to the manufacturer provided protocol, and daily phantom measurements were performed throughout the study. Both left and right hips were measured. Our analyses primarily include measures of the left hip; however, the right hip was used if measurements of the left side were missing or ineligible due to current or previous injuries or artifacts.

Precision of DXA measurements of total hip using the GE Lunar Prodigy has been evaluated in previous studies [31,32] which concluded that variation in one repeated aBMD measurement of less than $3 \%$ can be due to random measurement error. Hence, we defined least significant change (LSC) as 3\% of the mean aBMD value per sex and age group.

\section{Measures of physical capability and physical activity}

Experienced physiotherapists and nurses were trained, and they performed the physical capability testing, following the study protocol.

Grip strength was assessed following the Southampton protocol [33]. The Jamar Plus+ Digital Hand Dynamometer (Patterson Medical, Warrenville, IL, USA) was used, and three measurements for each hand were collected, alternating between right and left hand. The maximum result of the six trials was used in the analyses. Participants were seated in a chair holding the dynamometer and resting the arm at the chair's armrest, 90 degree angle at elbow, with the hand free from the armrest, thumb up. The dynamometer's position two (counted from display) of five possible settings was used for all participants.

The TUG test was performed according to the protocol by Podsiadlo and Richardson [34]. Participants were seated in a 43-cm-high chair with armrests and asked to stand up from the chair at the command "Go" (using the chair's armrests if they preferred), walk $3 \mathrm{~m}$ at their regular pace, turn and walk back again, and then sit down. The participant's score in seconds was timed with a stopwatch from the command "Go" to when the participant was seated again.

SPPB is composed of three subtests evaluating standing balance, walking speed, and repeated chair-stands. The SPPB was performed following the official Norwegian protocol $[35,36]$. The standing balance tests included tandem, semi-tandem, and side-by-side standing, and the participants were timed until they moved or $10 \mathrm{~s}$ had elapsed. To assess walking speed, the participants were timed while walking $4 \mathrm{~m}$ at their regular pace for two attempts. Before the five chairstands test, a pre-test was performed; the participants were asked to fold their arms across their chest (i.e., the armrests were not used) and stand up from the chair. If the pre-test was unsuccessful, the participant was scored zero. If the pre-test was successful, the participants were asked to perform five hair-stands as quickly as possible. They were timed (in seconds) from the initial sitting position to the final standing position at the fifth stand. Each of the three subtests of the SPPB were scored from zero to four and summed for a total score ranging from zero to 12 , with higher scores reflecting better function. Since a ceiling effect is likely to occur when relatively young and healthy participants perform the SPPB [35], we also utilized data from two of the sub-tests, namely the time spent to complete 5 chair-stands and to walk $4 \mathrm{~m}$ at regular pace as continuous, independent variables in separate analyses.

One-leg stance was performed in accordance with the protocol described by Kuh, D. et al. [37], where participants were asked to stand (with shoes) on the preferred leg with the foot of the other leg elevated off the floor a few inches. Participants were allowed to use their arms, bend their knees, or move the body to keep their balance and timed from when the foot was raised until they either moved their foot on which they were standing or put their elevated foot to the floor. They were timed for a maximum of $30 \mathrm{~s}$. The test was first performed with eyes open and then repeated with eyes closed. A clear ceiling effect was observed in the test with eyes open (63\% completed the maximum time of $30 \mathrm{~s}$ ), so the test results with eyes closed were chosen for the analyses ( $4 \%$ completed $30 \mathrm{~s}$, and $50 \%$ completed $3.5 \mathrm{~s}$ or less).

Level of physical activity was self-reported using the validated Saltin-Grimby Physical Activity Level Scale [38, 39] with four response categories: "Describe your exercise and physical exertion in leisure time the last year: 1) Reading, watching TV/screen or other sedentary activity. 2) Walking, cycling or other forms of exercise at least 4 hours a week (including walking or cycling to place of work, Sunday-walking, etc.) 3) Participation in recreational sports, heavy gardening, snow shoveling etc. at least 4 hours a week. 4) Participation in hard training or sports competitions regularly several times a week."

\section{Covariates}

Height and weight were measured by trained personnel while wearing light clothes and without shoes. BMI was calculated as weight in kilograms divided by height in 
Table 1 Characteristics of women and men by age groups. Continuous variables presented as mean values with standard deviations (SD) and categorical variables presented as number of individuals and percent

\begin{tabular}{|c|c|c|c|c|c|c|c|c|}
\hline & \multicolumn{4}{|c|}{ Women $(N=2052)$} & \multicolumn{4}{|c|}{$\operatorname{Men}(N=1481)$} \\
\hline & \multicolumn{2}{|c|}{$\leq 65(N=903)$} & \multicolumn{2}{|c|}{$>65(N=1149)$} & \multicolumn{2}{|c|}{$\leq 65(N=665)$} & \multicolumn{2}{|c|}{$>65(N=816)$} \\
\hline & Mean & $\mathrm{SD}$ & Mean & $\mathrm{SD}$ & Mean & $\mathrm{SD}$ & Mean & SD \\
\hline Age (years) & 58.0 & 6.5 & 72.2 & 4.5 & 57.9 & 6.7 & 72.0 & 4.8 \\
\hline Height $(\mathrm{cm})$ & 164.6 & 6.2 & 162.0 & 6.0 & 178.0 & 6.7 & 175.3 & 6.4 \\
\hline Weight $(\mathrm{kg})$ & 71.2 & 13.0 & 71.4 & 12.8 & 87.9 & 14.1 & 84.4 & 12.0 \\
\hline $\mathrm{BMI}\left(\mathrm{kg} / \mathrm{m}^{2}\right)$ & 26.3 & 4.6 & 27.2 & 4.7 & 27.7 & 3.9 & 27.4 & 3.5 \\
\hline aBMD total hip $\left(\mathrm{g} / \mathrm{cm}^{2}\right)$ & .932 & .130 & .886 & .130 & 1.060 & .134 & 1.030 & .159 \\
\hline Grip strength $(\mathrm{kg})$ & 29.6 & 4.9 & 25.8 & 4.8 & 51.2 & 8.4 & 43.2 & 7.8 \\
\hline TUG total score & 7.9 & 2.7 & 9.2 & 2.3 & 7.9 & 1.4 & 9.1 & 2.0 \\
\hline SPPB & 11.6 & 1.1 & 10.8 & 1.7 & 11.8 & .8 & 11.2 & 1.4 \\
\hline Chair-stand (s) & 9.2 & 3.6 & 11.1 & 3.2 & 8.4 & 2.3 & 10.0 & 3.3 \\
\hline $4 \mathrm{~m}$ walk (s) & 3.4 & .7 & 3.9 & 1.0 & 3.3 & .6 & 3.8 & .9 \\
\hline \multirow[t]{2}{*}{ One-leg stance (s) } & 8.8 & 8.8 & 3.7 & 4.2 & 8.9 & 8.7 & 4.2 & 4.4 \\
\hline & $\mathrm{N}$ & $\%$ & $\mathrm{~N}$ & $\%$ & $\mathrm{~N}$ & $\%$ & $\mathrm{~N}$ & $\%$ \\
\hline \multicolumn{9}{|l|}{ Smoking } \\
\hline Never & 333 & 36.9 & 498 & 43.3 & 274 & 41.2 & 251 & 30.8 \\
\hline Previous & 430 & 47.6 & 542 & 47.2 & 311 & 46.8 & 499 & 61.2 \\
\hline Current & 140 & 15.5 & 109 & 9.5 & 80 & 12.0 & 66 & 8.1 \\
\hline \multicolumn{9}{|l|}{ Education level* } \\
\hline 1 & 261 & 28.9 & 173 & 15.1 & 167 & 25.1 & 118 & 14.5 \\
\hline 2 & 180 & 19.9 & 128 & 11.1 & 141 & 21.2 & 179 & 21.9 \\
\hline 3 & 255 & 28.2 & 284 & 24.7 & 201 & 30.2 & 239 & 29.3 \\
\hline 4 & 207 & 22.9 & 564 & 49.1 & 156 & 23.5 & 280 & 34.3 \\
\hline \multicolumn{9}{|l|}{ Diseases** } \\
\hline Cardiovascular & 259 & 28.7 & 568 & 49.4 & 260 & 39.1 & 483 & 32.6 \\
\hline High BP & 228 & 25.2 & 503 & 43.8 & 209 & 31.4 & 363 & 24.5 \\
\hline Heart attack & 8 & .9 & 45 & 3.9 & 24 & 3.6 & 97 & 6.5 \\
\hline Heart failure & 5 & .6 & 25 & 2.2 & 14 & 2.1 & 50 & 3.4 \\
\hline Atrial fibrillation & 32 & 3.5 & 95 & 8.3 & 42 & 6.3 & 109 & 7.4 \\
\hline Angina & 7 & .8 & 35 & 3.0 & 16 & 2.4 & 48 & 3.2 \\
\hline Stroke & 13 & 1.4 & 35 & 3.0 & 16 & 2.4 & 49 & 3.3 \\
\hline Metabolic & 65 & 7.2 & 122 & 10.6 & 39 & 5.9 & 103 & 7.0 \\
\hline Diabetes & 40 & 4.4 & 77 & 6.7 & 29 & 4.4 & 67 & 4.5 \\
\hline Kidney disease & 28 & 3.1 & 47 & 4.1 & 14 & 2.1 & 42 & 2.8 \\
\hline Respiratory & 119 & 13.2 & 189 & 16.4 & 62 & 9.3 & 107 & 7.2 \\
\hline Bronchitis & 33 & 3.7 & 64 & 5.6 & 13 & 2.0 & 40 & 2.7 \\
\hline Asthma & 106 & 11.7 & 152 & 13.2 & 53 & 8.0 & 76 & 5.1 \\
\hline Cancer & 68 & 7.5 & 153 & 13.3 & 47 & 7.1 & 128 & 8.6 \\
\hline Musculoskeletal & 250 & 27.7 & 526 & 45.8 & 97 & 14.6 & 175 & 11.8 \\
\hline Rheumatoid arthritis & 42 & 4.7 & 87 & 7.6 & 29 & 4.4 & 36 & 2.4 \\
\hline Arthrosis & 230 & 25.5 & 487 & 42.4 & 77 & 11.6 & 152 & 10.3 \\
\hline \multicolumn{9}{|l|}{ Physical activity level**** } \\
\hline 1 & 100 & 11.2 & 123 & 11.6 & 71 & 10.8 & 115 & 14.7 \\
\hline 2 & 610 & 68.5 & 758 & 71.4 & 376 & 57.1 & 433 & 55.2 \\
\hline 3 & 162 & 18.2 & 171 & 16.1 & 188 & 28.6 & 228 & 29.1 \\
\hline 4 & 19 & 2.1 & 9 & 8 & 23 & 3.5 & 8 & 1.0 \\
\hline
\end{tabular}

*Education level 1: primary/partly secondary education (10 years), 2: upper secondary education (10-12 years), 3: tertiary education short: college/ university (13-15 years), 4: tertiary education long: college/university (16+ years)

**Number of participants who report having given disease, either currently or previously diagnosed

***Physical exertion in leisure time: 1: "Reading, watching TV/screen or other sedentary activity." 2: "Walking, cycling or other forms of exercise at least 4 hours a week (including walking or cycling to place of work, Sunday-walking, etc.)," 3: "Participation in recreational sports, heavy gardening, snow shoveling etc. at least 4 hours a week," 4: "Participation in hard training or sports competitions regularly several times a week"

$N$ number of participants, $S D$ standard deviation, $B M I$ body mass index, $a B M D$ areal bone mineral density, $T U G$ Timed Up and Go, SPPB Short Physical Performance Battery, $B P$ blood pressure

meters squared $\left(\mathrm{kg} / \mathrm{m}^{2}\right)$ and used as a continuous variable in the analyses. Daily smoking was self-reported as "yes now," "yes previously," or "never-smoker." Highest attained education level was self-reported with the following categories: (1) primary/partly secondary education (10 years); (2) upper secondary education (10-12 years); (3) tertiary 
Fig. 1 Flow chart illustrating inclusion and exclusion of participants

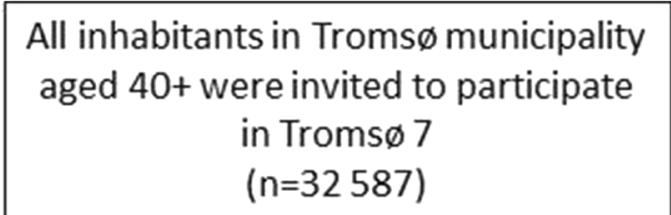

Declined participation ( $n=11504)$

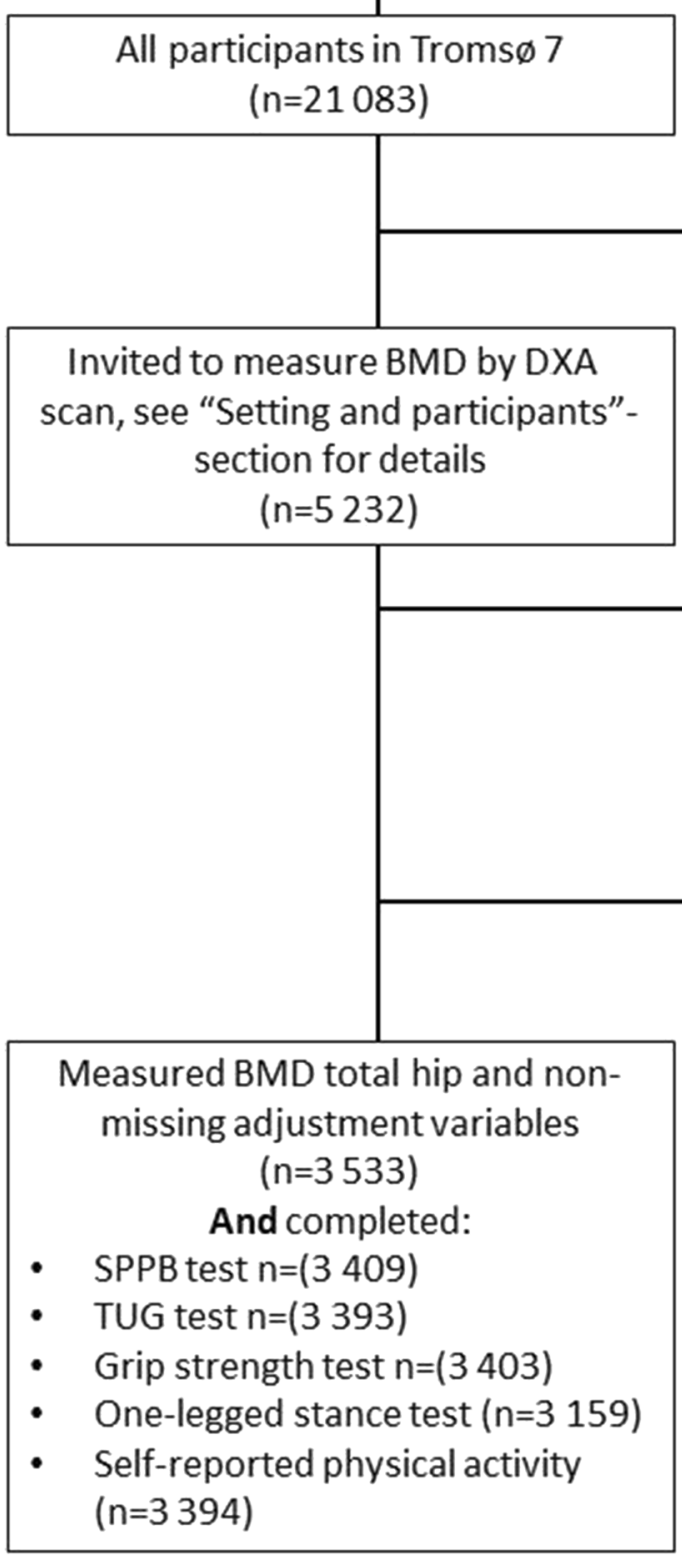

Not invited to measure BMD by

DXA scan

$(n=15851)$
Declined/were not able to participate/did not complete DXA measurements in total hip $(n=1574)$

\section{Missing values for one or more} adjustment variables $(n=125)$ education short: college/university (13-15 years); and (4) tertiary education long: college/university (16+ years). Self-reported current or previous chronic diseases were registered if the participant replied "yes currently" or "previously" to questions about hypertension, myocardial infarction, heart failure, atrial fibrillation, angina, stroke, diabetes, kidney disease, bronchitis, asthma, cancer, rheumatoid arthritis, or osteoarthritis in their medical history. Diseases were categorized as "cardiovascular disease," "metabolic disease," "respiratory disease," "cancer," or "musculoskeletal 
disease" in the analyses; see Table 1 for overview of the disease categories.

\section{Least significant change}

More than 3\% difference is required for an aBMD measurement to be significantly different from a previous measurement in the same individual, using the GE Lunar Prodigy $[31,32]$. For physical capability tests that were significantly associated with aBMD, test score difference corresponding to $3 \%$ aBMD difference was calculated.

Table 2 Regression coefficients with 95\% confidence intervals for change in total hip aBMD $\left(\mathrm{g} / \mathrm{cm}^{2}\right)$ per unit change in grip strength, Timed Up and Go (TUG), Short Physical Performance Battery (SPPB)

\section{Statistical analysis}

The association between the continuous outcome variable (aBMD) and the continuous physical capability variables, as well as the categorical physical activity variable, was examined individually using multiple linear regressions. All analyses were performed separately by sex. Model 1 was adjusted for age, while model 2 included additional adjustment for BMI ( $\mathrm{kg} / \mathrm{m}^{2}$, continuous), height ( $\mathrm{cm}$, continuous), daily smoking (categorical), education (categorical), and all listed chronic disease groups added separately to the model (categorical). In addition to models including all ages, separate models for participants aged $\leq 65$ years and $>65$ years were performed, enabling comparison with previous studies.

including total score and time spent on the $5 \times$ sit-to-stand test, and $4 \mathrm{~m}$ walk test, one-leg stance (OLS) with eyes closed, and self-reported level of physical activity in women, overall and stratified by age at 65 years

\begin{tabular}{|c|c|c|c|c|c|c|c|c|c|c|}
\hline \multicolumn{11}{|l|}{ Women } \\
\hline \multirow{4}{*}{$\begin{array}{l}\text { Exposure variable } \\
\text { Grip strength (kg) }\end{array}$} & Age (years) & Number & $\begin{array}{l}\text { Regression } \\
\text { coefficient }\end{array}$ & \multicolumn{2}{|c|}{$\begin{array}{l}\text { Age-adjusted } \\
95 \% \text { CI }\end{array}$} & \multirow{2}{*}{$\begin{array}{l}Z \text {-score } \\
.11\end{array}$} & \multirow{2}{*}{$\begin{array}{l}\text { Regression } \\
\text { coefficient } \\
* .001\end{array}$} & \multicolumn{2}{|c|}{$\begin{array}{l}\text { Fully adjusted** } \\
95 \% \text { CI }\end{array}$} & \multirow{2}{*}{$\begin{array}{l}\text { Z-score } \\
.05\end{array}$} \\
\hline & All & 1980 & $* .003$ & .002 & .004 & & & .000 & .002 & \\
\hline & $\leq 65$ & 871 & $* .003$ & .001 & .005 & .12 & .001 & -.001 & .003 & .04 \\
\hline & $>65$ & 1109 & $* .003$ & .001 & .004 & .10 & .002 & -.000 & .003 & .06 \\
\hline TUG (s) & All & 1975 & -.000 & -.003 & .002 & -.01 & $*-.004$ & -.006 & -.001 & -.07 \\
\hline & $\leq 65$ & 868 & .001 & -.002 & .004 & .01 & -.001 & -.003 & .002 & -.01 \\
\hline & $>65$ & 1107 & -.002 & -.005 & .002 & -.03 & $*-.007$ & -.010 & -.004 & -.13 \\
\hline \multicolumn{11}{|l|}{ SPPB } \\
\hline \multirow[t]{3}{*}{ Total score $(0-12)$} & All & 1986 & .003 & -.001 & .007 & .04 & $* .009$ & .005 & .012 & .10 \\
\hline & $\leq 65$ & 873 & .007 & -.001 & .015 & .05 & $* .010$ & .003 & .017 & .08 \\
\hline & $>65$ & 1113 & .002 & -.003 & .007 & .03 & $* .009$ & .005 & .013 & .12 \\
\hline \multirow[t]{3}{*}{ Chair- stand test (s) } & All & 1954 & .000 & -.002 & .002 & .00 & $*-.002$ & -.004 & -.001 & -.07 \\
\hline & $\leq 65$ & 867 & -.000 & -.003 & .002 & -.00 & -.002 & -.004 & .001 & -.05 \\
\hline & $>65$ & 1087 & .000 & -.002 & .003 & .00 & $*-.004$ & -.006 & -.001 & -.09 \\
\hline \multirow[t]{3}{*}{ Time 4 m walk (s) } & All & 1980 & -.005 & -.011 & .002 & -.03 & $*-.013$ & -.019 & -.007 & -.09 \\
\hline & $\leq 65$ & 869 & -.012 & -.024 & .001 & -.06 & $*-.022$ & -.033 & -.010 & -.11 \\
\hline & $>65$ & 1111 & -.002 & -.010 & .006 & -.02 & $*-.011$ & -.018 & -.003 & -.08 \\
\hline \multirow[t]{3}{*}{ One-leg stance (s, eyes closed) } & All & 1826 & -.000 & -.001 & .000 & -.02 & $* .001$ & .001 & .002 & .08 \\
\hline & $\leq 65$ & 861 & -.000 & -.001 & .001 & -.02 & $* .002$ & .001 & .003 & .10 \\
\hline & $>65$ & 965 & -.001 & -.003 & .001 & -.04 & .001 & -.001 & .003 & .04 \\
\hline \multicolumn{11}{|l|}{ Physical activity } \\
\hline $\begin{array}{r}* * * \text { Level: } \\
2\end{array}$ & All & 1952 & .013 & -.004 & .031 & - & $* .037$ & .021 & .053 & - \\
\hline 3 & & & .018 & -.003 & .040 & - & $* .049$ & .030 & .069 & - \\
\hline 4 & & & .048 & -.002 & .098 & - & $* .093$ & .048 & .137 & - \\
\hline 2 & $\leq 65$ & 891 & .022 & -.004 & .048 & - & $* .039$ & .015 & .063 & - \\
\hline 3 & & & .017 & -.014 & .048 & - & $* .047$ & .019 & .075 & - \\
\hline 4 & & & $* .063$ & .001 & .125 & - & $* .104$ & .049 & .160 & - \\
\hline 2 & $>65$ & 1061 & .003 & -.021 & .027 & - & $* .035$ & .012 & .057 & - \\
\hline 3 & & & .016 & -.013 & .046 & - & $* .051$ & .024 & .078 & - \\
\hline 4 & & & .003 & -.083 & .089 & - & .062 & -.015 & .139 & - \\
\hline
\end{tabular}

* Statistically significant results $(p<0.05)$

**Fully adjusted model: adjusted for age, body mass index, smoking, education, height, and comorbidities

***Level of physical activity compared to level 1: "Reading, watching TV/screen or other sedentary activity." Level 2 described as "Walking, cycling or other forms of exercise at least 4 hours a week (including walking or cycling to place of work, Sunday-walking, etc.)," level 3: "Participation in recreational sports, heavy gardening, snow shoveling etc. at least 4 hours a week," and level 4: "Participation in hard training or sports competitions regularly several times a week"

$N$ number of participants, $C I$ confidence interval, $T U G$ Timed Up and Go, SPPB Short Physical Performance Battery

The Z-score column represents the effect size when both the outcome and predictor are standardized 
Table 3 Regression coefficients with 95\% confidence intervals for change in total hip aBMD $\left(\mathrm{g} / \mathrm{cm}^{2}\right)$ per unit change in grip strength, Timed Up and Go (TUG), Short Physical Performance Battery (SPPB) including total score and time spent on the $5 \times$ sit-to-stand test, and $4 \mathrm{~m}$ walk test, one-leg stance (OLS) with eyes closed, and self-reported level of physical activity in men, overall and stratified by age at 65 years

Men

\begin{tabular}{|c|c|c|c|c|c|c|c|c|c|c|}
\hline \multirow{3}{*}{$\begin{array}{l}\text { Exposure variable } \\
\text { Grip strength }(\mathrm{kg})\end{array}$} & \multirow{3}{*}{$\begin{array}{l}\text { Age } \\
\text { All }\end{array}$} & \multirow{3}{*}{$\begin{array}{l}\text { Number } \\
1423\end{array}$} & \multicolumn{4}{|l|}{ Age-adjusted } & \multicolumn{4}{|l|}{ Fully adjusted** } \\
\hline & & & \multirow{2}{*}{$\begin{array}{l}\text { Regression coefficient } \\
* 002\end{array}$} & \multicolumn{2}{|l|}{$95 \% \mathrm{CI}$} & \multirow{2}{*}{$\begin{array}{l}\begin{array}{l}\mathrm{Z}- \\
\text { score }\end{array} \\
.12\end{array}$} & \multirow{2}{*}{$\begin{array}{l}\text { Regression coefficient } \\
.001\end{array}$} & \multicolumn{2}{|l|}{$95 \% \mathrm{CI}$} & \multirow{2}{*}{$\begin{array}{l}\text { Z-score } \\
.05\end{array}$} \\
\hline & & & & .001 & .003 & & & -.000 & .002 & \\
\hline & $\leq 65$ & 636 & $* .002$ & .000 & .003 & .10 & .001 & -.001 & .002 & .04 \\
\hline & $>65$ & 787 & $* .002$ & .001 & .004 & .11 & .001 & -.000 & .003 & .06 \\
\hline \multirow[t]{3}{*}{ TUG (s) } & All & 1418 & -.002 & -.007 & .002 & -.03 & -.004 & -.008 & .001 & .05 \\
\hline & $\leq 65$ & 635 & .001 & -.006 & .009 & .01 & -.002 & -.009 & .006 & -.02 \\
\hline & $>65$ & 783 & -.003 & -.009 & .003 & -.04 & -.004 & -.010 & .003 & -.04 \\
\hline \multicolumn{11}{|l|}{ SPPB } \\
\hline \multirow[t]{3}{*}{ Total score $(0-12)$} & All & 1423 & $* .007$ & .000 & .014 & .06 & $* .011$ & .004 & .017 & .09 \\
\hline & $\leq 65$ & 636 & -.009 & -.021 & .004 & -.05 & -.003 & -.015 & .009 & -.02 \\
\hline & $>65$ & 851 & $* .011$ & .002 & .019 & .09 & $* .014$ & .006 & .023 & .12 \\
\hline \multirow[t]{3}{*}{ Chair- stand test (s) } & All & 1412 & $*-.003$ & -.006 & -.001 & -.07 & $*-.005$ & -.008 & -.002 & -.10 \\
\hline & $\leq 65$ & 633 & -.001 & -.005 & .004 & .01 & -.003 & -.008 & .001 & -.06 \\
\hline & $>65$ & 779 & $*-.004$ & -.008 & -.001 & -.09 & $*-.006$ & -.009 & -.002 & -.11 \\
\hline \multirow[t]{3}{*}{ Time 4 m walk (s) } & All & 1420 & $*-.011$ & -.021 & -.001 & -.06 & $*-.011$ & -.021 & -.001 & -.06 \\
\hline & $\leq 65$ & 635 & -.008 & -.025 & .009 & -.04 & -.005 & -.021 & .012 & -.02 \\
\hline & $>65$ & 785 & -.010 & -.024 & .003 & -.06 & -.011 & -.024 & .002 & -.06 \\
\hline \multirow[t]{3}{*}{ One-leg stance (s, eyes closed) } & All & 1333 & -.000 & -.001 & .001 & -.01 & .001 & -.000 & .002 & .04 \\
\hline & $\leq 65$ & 628 & -.000 & -.001 & .001 & -.00 & .001 & -.001 & .002 & .04 \\
\hline & $>65$ & 705 & -.000 & -.003 & .003 & .00 & .001 & -.002 & .004 & .03 \\
\hline \multicolumn{11}{|l|}{ Physical activity } \\
\hline \multirow[t]{9}{*}{ ***Level: } & 2 & All & 1442 & .016 & -.008 & .039 & - & .021 & -.001 & .044 \\
\hline & 3 & & & $* .034$ & .008 & .059 & - & $* .045$ & .020 & .070 \\
\hline & 4 & & & -.003 & -.059 & .053 & - & .018 & -.036 & .072 \\
\hline & 2 & $\leq 65$ & 658 & .003 & -.031 & .036 & - & .011 & -.021 & .044 \\
\hline & 3 & & & .033 & -.003 & .069 & - & $* .047$ & .012 & .083 \\
\hline & 4 & & & .008 & -.054 & .069 & - & .036 & -.024 & .096 \\
\hline & 2 & $>65$ & 784 & .028 & -.004 & .061 & - & $* .032$ & .001 & .064 \\
\hline & 3 & & & .034 & -.001 & .069 & - & $* .044$ & .009 & .078 \\
\hline & 4 & & & -.044 & -.157 & .068 & - & -.030 & -.139 & .079 \\
\hline
\end{tabular}

*Statistically significant results $(p<0.05)$

**Fully adjusted model: adjusted for age, body mass index, smoking, education, height, and comorbidities

***Level of physical activity compared to level 1: "Reading, watching TV/screen or other sedentary activity." Level 2 described as: "Walking, cycling or other forms of exercise at least 4 hours a week (including walking or cycling to place of work, Sunday-walking, etc.)," level 3: "Participation in recreational sports, heavy gardening, snow shoveling etc. at least 4 hours a week," and level 4: "Participation in hard training or sports competitions regularly several times a week"

$N$ number of participants, $C I$ confidence interval, $T U G$ Timed Up and Go, SPPB Short Physical Performance Battery

The Z-score column represents the effect size when both the outcome and predictor are standardized

To investigate possible non-linear associations, the fully adjusted regression analyses, with aBMD as a continuous outcome variable, were also modeled with cubic splines of the continuous physical capability variables, with 4 knots at default knot location and 95\% confidence intervals (CI). The association between aBMD and SPPB total score could not be modeled with splines due to a strong ceiling effect. For analyses of physical activity, predicted mean aBMD with 

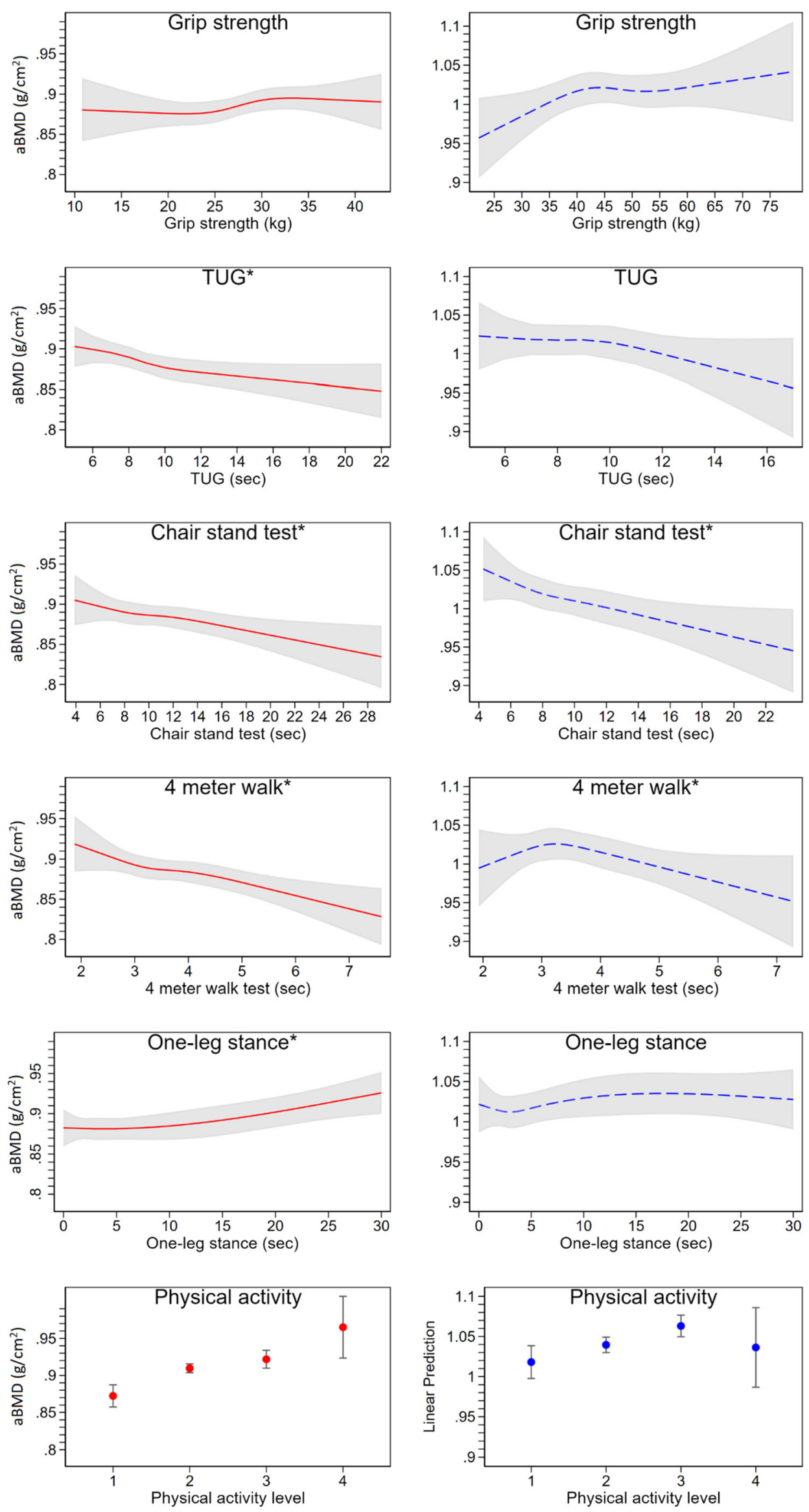
Fig. 2 Association between aBMD total hip and physical performance test results with $95 \%$ confidence intervals in women (solid line/red) and men (dashed line/blue) aged 40-84 years. *Test of overall association $p<$ 0.05 (based on a likelihood ratio test comparing the models with and without the splines). For physical activity, the models compared are the ones with and without the physical activity variable

95\% CI was plotted according to categories of physical activity level. In addition to the regression analysis entering aBMD in $\mathrm{g} / \mathrm{cm}^{2}$ and the continuous exposure variables on their original scales, a regression analysis was performed entering standardized values of both the outcome variable (aBMD) and the physical capability predictor variables expressed as Z-scores calculated by sex, to obtain standardized coefficients and thus comparable effect sizes across the physical capability tests. Coefficient differences between sex and age groups were tested using the Chow test. Statistical significance was determined by a $p$-value $<$ 0.05. All analyses were carried out using Stata version 15.0.

\section{Ethical approval}

The Tromsø study, as well as our research project, is approved by the Regional Committees for Medical and Health Research Ethics in Norway (REC). All participants in The Tromsø study have received an information letter and given written consent prior to participation in the study.

\section{Results}

Table 1 shows values of background characteristics and scores of the performance-based physical capability tests (grip strength, TUG, SPPB, chair-stands, walking speed, and oneleg stance) of 3522 participants, as well as values of physical activity of women and men by age groups. Better performance on all the individual tests of physical capability was associated with significantly higher aBMD in women (Table 2). In men, however, only SPPB, chair-stands, and walking speed were associated with aBMD in the fully adjusted models (Table 3 ). No tests were significantly associated with aBMD in the younger men in the age stratified analyses.

Physical activity was significantly associated with aBMD in both women and men and in both age groups in the fully adjusted models. While physical activity level 3 was associated with significantly higher aBMD compared to level 1 (sedentary) in both women and men, level 2 was also associated with significantly higher aBMD than level 1 in all female age groups and in older men. The highest level of physical activity (level 4) was associated with the highest aBMD in women 65 years old and younger. See Tables 2 and 3 for the complete overview of regression coefficients.

Based on the analyses of the physical capability test results expressed as Z-scores (Tables 2 and 3), the strongest association with total hip aBMD was observed for the SPPB total score in women (standardized regression coefficient: 0.10 ) and chairstand test in men (standardized regression coefficient: -0.10 ). In younger women, the $4 \mathrm{~m}$ walk test gave the strongest association with aBMD (standardized regression coefficient: -0.11 ), while TUG gave the strongest association for older women
Table 4 Number of units change corresponding to least detectable aBMD change $(3 \%)$ in women and men. Numbers given for continuous variables significantly associated with aBMD in the fully adjusted models. For physical activity, percent aBMD increase is given per level compared to level 1 (lowest)

\begin{tabular}{|c|c|c|c|c|c|c|}
\hline \multirow[t]{3}{*}{$3 \%$ of average aBMD $\left(\mathrm{g} / \mathrm{cm}^{2}\right)$} & \multicolumn{3}{|c|}{ Women: $3 \%$ aBMD change } & \multicolumn{3}{|c|}{ Men: $3 \%$ aBMD change } \\
\hline & All & $\leq 65$ & $>65$ & All & $\leq 65$ & $>65$ \\
\hline & 0.027 & 0.028 & 0.027 & 0.031 & 0.032 & 0.031 \\
\hline Grip strength (kg) & 22.0 & - & - & - & - & - \\
\hline TUG (s) & 7.6 & - & 3.8 & - & - & - \\
\hline SPPB total score (1-12) & 3.1 & 2.8 & 3.0 & 2.9 & - & 2.2 \\
\hline Chair-stand test (s) & 11.0 & - & 7.4 & 6.1 & - & 5.6 \\
\hline 4 m walk (s) & 2.1 & 1.3 & 2.6 & 2.9 & - & - \\
\hline One-leg stance (s, $\max 30)$ & 18.8 & 18.2 & - & - & - & - \\
\hline Physical activity level* & \multicolumn{6}{|c|}{$\%$ aBMD increase compared to activity level 1} \\
\hline 2 & $4.1 \%$ & $4.2 \%$ & $4.0 \%$ & - & - & $3.1 \%$ \\
\hline 3 & $5.5 \%$ & $5.0 \%$ & $5.8 \%$ & $4.3 \%$ & $4.4 \%$ & $4.3 \%$ \\
\hline 4 & $10.2 \%$ & $11.2 \%$ & - & - & - & - \\
\hline
\end{tabular}

*Physical activity level 1: reading, watching TV/screen or other sedentary activity, 2 : walking, cycling or other forms of exercise at least $4 \mathrm{~h}$ a week (including walking or cycling to place of work, Sunday-walking, etc.), 3: participation in recreational sports, heavy gardening, snow shoveling, etc. at least $4 \mathrm{~h}$ a week, 4: participation in hard training or sports competitions regularly several times a week

$a B M D$ areal bone mineral density, $T U G$ Timed Up and Go, SPPB Short Physical Performance Battery 
(standardized regression coefficients: - 0.13). Self-reported physical activity as a categorical variable was significantly associated with aBMD in all sex and age groups. No statistically significant associations were found between aBMD and any of the continuous measurements of physical capability in younger men; hence, level of physical activity was the best predictor of aBMD in this group. In the older men, SPPB total score was the strongest predictor of aBMD (standardized regression coefficient 0.12 ).

There were no statistically significant differences between men and women within the same age groups. There was however a significant difference in effect of TUG on aBMD between young and old women $(p=0.002)$ and SPPB total score and $\mathrm{aBMD}$ in young and old men $(p=0.028)$. Associations between aBMD and tests of physical capability modeled with cubic splines are presented for women and men in Fig. 2.

\section{Clinical relevance of aBMD difference}

Table 4 presents number of unit difference corresponding to $1 \times$ LSC in aBMD among the continuous variables. If the relationships between physical performance tests and aBMD are causal, a woman would need to improve grip strength with $22 \mathrm{~kg}$, TUG execution time with $7.6 \mathrm{~s}$, SPPBscore with 3.1 points, chair-stand test time with $11 \mathrm{~s}, 4 \mathrm{~m}$ walk test with $2.1 \mathrm{~s}$, or one-leg stance with $18.8 \mathrm{~s}$ in order to achieve $1 \times$ LSC in aBMD. For men, in whom we found statistically significant associations between the tests included in the SPPB and aBMD, chair-stand test time would need to be improved with $6.1 \mathrm{~s}$, otherwise the numbers are similar to those in women.

\section{Discussion}

This large population-based study found a positive relationship between physical activity and physical capability and total hip aBMD in a general adult population. Leisure time physical activity level was strongly positively associated with aBMD of the total hip in both women and men. All the individual physical capability tests included in our study were positively associated with total hip aBMD in women in the fully adjusted models. For men, statistically significant associations were found between aBMD and the tests SPPB, chairstands, and walking speed.

For women and men, both younger and older than 65 years, physical activity was significantly associated with total hip aBMD. This finding underlines the importance for both women and men to be physically active through adulthood and in older age in order to maintain good bone health. Even low-intensity activities like "walking, cycling or other forms of exercise at least 4 hours a week" were beneficial for total hip aBMD in women and in older men compared to a sedentary lifestyle. A total of 59 out of 3 394 participants reported the highest level of physical activity ("Participation in hard training or sports competitions regularly several times a week"). Although we found a statistically significant higher aBMD in those reporting the highest level of physical activity in the younger women, the overall proportion who reported this activity level was low, and we cannot infer with certainty whether such a high level of physical activity is beneficial for total hip aBMD.

Grip strength was associated with total hip aBMD in women and men in both age groups in the age-adjusted models, but only in women with both age groups combined in the fully adjusted models. While we and others have found grip strength to be associated with hip fractures and other osteoporotic fractures $[24,26,40]$, it is possible that grip strength is more closely related to the risk of falling than it is to osteoporosis. Other studies have indicated that grip strength is associated with upper limb BMD [18] which we did not assess in our study. While grip strength may be a strong predictor of fractures, it does not appear to be a strong predictor of lower limb aBMD.

In general, the relationship between physical capability and total hip aBMD was similar in women and men in our study; thus, our results seem to be at odds with those reported by Shin $\mathrm{H}$ et al. [18] who found a stronger relationship between physical capability and BMD in women. However, even if we found no significant sex by capability interaction, results were more often significant in women than in men. This is possibly due to the substantially larger sample of women than men in our study and also reflects that a relatively large sample size was needed in order to detect a statistically significant relationship between physical capability and total hip aBMD.

This study was based on cross-sectional data and can therefore not reveal causal relationships between physical capability and physical activity and total hip aBMD. Well-designed and updated RCTs are needed to be able to determine if targeted training to improve physical capability and increased leisure time physical activity give any aBMD benefits.

Even though we found statistically significant associations between physical capability and total hip aBMD, the effect sizes were small and the clinical significance may be limited. The differences in test performance corresponding to a least significant aBMD change (3\%), theoretically indicating the improvements in capability required to achieve an increase in aBMD, are substantial and unfeasible for most individuals. Meanwhile, improving level of physical activity from sedentary (level 1) to light physical activity (level 2) would correspond to more than $3 \%$ higher aBMD in women and in older men. Although such a change would require persisting motivation and long-term behavioral change, we believe that it is feasible to achieve for most people. 
However, in a population where age-related bone loss is inevitable, focus should be on limiting the bone loss rather than aiming to increase aBMD. Our results show that physical capability is positively associated with total hip aBMD and indicate the importance of maintaining one's physical capability since a poorer score is associated with a decrease in aBMD.

\section{Conclusions}

Physical activity was positively associated with total hip aBMD in both younger and older women and men. In addition, better results on the physical capability tests TUG, SPPB, chair-stands, walking speed, and balance were associated with higher total hip aBMD in women, while SPPB, chair-stands, and walking speed were associated with aBMD in men. Although increasing aBMD is not likely, our findings suggest that maintaining a high level of physical capability and activity might be helpful in preventing bone loss through midlife and older age.

Funding Open access funding provided by OsloMet - Oslo Metropolitan University.

\section{Declarations}

Competing interests The authors declare no competing interests.

Open Access This article is licensed under a Creative Commons Attribution-NonCommercial 4.0 International License, which permits any non-commercial use, sharing, adaptation, distribution and reproduction in any medium or format, as long as you give appropriate credit to the original author(s) and the source, provide a link to the Creative Commons licence, and indicate if changes were made. The images or other third party material in this article are included in the article's Creative Commons licence, unless indicated otherwise in a credit line to the material. If material is not included in the article's Creative Commons licence and your intended use is not permitted by statutory regulation or exceeds the permitted use, you will need to obtain permission directly from the copyright holder. To view a copy of this licence, visit http:// creativecommons.org/licenses/by-nc/4.0/.

\section{References}

1. Cianferotti L, Brandi ML (2014) Muscle-bone interactions: basic and clinical aspects. Endocrine 45(2):165-177

2. Ho-Pham LT, Nguyen UD, Nguyen TV (2014) Association between lean mass, fat mass, and bone mineral density: a metaanalysis. J Clin Endocrinol Metab 99(1):30-38

3. Blain H, Jaussent A, Thomas E, Micallef JP, Dupuy AM, Bernard PL, Mariano-Goulart D, Cristol JP, Sultan C, Rossi M, Picot MC (2010) Appendicular skeletal muscle mass is the strongest independent factor associated with femoral neck bone mineral density in adult and older men. Exp Gerontol 45(9):679-684
4. Kim S, Won CW, Kim BS, Choi HR, Moon MY (2014) The association between the low muscle mass and osteoporosis in elderly Korean people. J Korean Med Sci 29(7):995-1000

5. Genaro PS, Pereira GAP, Pinheiro MM, Szejnfeld VL, Martini LA (2010) Influence of body composition on bone mass in postmenopausal osteoporotic women. Arch Gerontol Geriatr 51(3):295-298

6. Demontiero O, Vidal C, Duque G (2012) Aging and bone loss: new insights for the clinician. Therapeut Adv Musculoskelet Dis 4(2): 61-76

7. Cruz-Jentoft AJ, Sayer AA (2019) Sarcopenia. Lancet 393:26362646

8. Reginster J-Y, Beaudart C, Buckinx F, Bruyère O (2016) Osteoporosis and sarcopenia: two diseases or one? Curr Opin Clin Nutr Metab Care 19(1):31-36

9. Howe TE, Shea B, Dawson LJ, Downie F, Murray A, Ross C, Harbour RT, Caldwell LM, Creed G (2011) Exercise for preventing and treating osteoporosis in postmenopausal women. Cochrane Database of Systematic Reviews 2011, Issue 7. Art. No.: CD000333. https://doi.org/10.1002/14651858.CD000333.pub2

10. McMillan LB, Zengin A, Ebeling PR, Scott D (2017) Prescribing Physical Activity for the Prevention and Treatment of Osteoporosis in Older Adults. Healthcare 5(4):85. https://doi.org/10.3390/ healthcare 5040085

11. Segev D, Hellerstein D, Dunsky A (2018) Physical activity-does it really increase bone density in postmenopausal women? A review of articles published between 2001-2016. Curr Aging Sci 11(1):4-9

12. Morseth B, Emaus N, Jørgensen L (2011) Physical activity and bone: the importance of the various mechanical stimuli for bone mineral density. A review. Norsk Epidemiol 20(2):173-178

13. Abrahin O, Rodrigues RP, Marçal AC, Alves EAC, Figueiredo RC, Sousa EC (2016) Swimming and cycling do not cause positive effects on bone mineral density: a systematic review. Rev Bras Reumatol 56(4):345-351

14. Simas V, Hing W, Pope R, Climstein M (2017) Effects of waterbased exercise on bone health of middle-aged and older adults: a systematic review and meta-analysis. Open Access J Sports Med 8: 39-60

15. Muir JM, Ye C, Bhandari M, Adachi JD, Thabane L (2013) The effect of regular physical activity on bone mineral density in postmenopausal women aged 75 and over: a retrospective analysis from the Canadian multicentre osteoporosis study. BMC Musculoskelet Disord 14(1):253

16. Coupland C et al (1999) Habitual physical activity and bone mineral density in postmenopausal women in England. Int J Epidemiol 28(2):241-246

17. Kuh D, Karunananthan S, Bergman H, Cooper R (2014) A lifecourse approach to healthy ageing: maintaining physical capability. Proc Nutr Soc 73(2):237-248

18. Shin H, Panton LB, Dutton GR, Ilich JZ (2011) Relationship of physical performance with body composition and bone mineral density in individuals over 60 years of age: a systematic review. $\mathrm{J}$ Aging Res 2011:1-14

19. Taaffe DR, Cauley JA, Danielson M, Nevitt MC, Lang TF, Bauer DC, Harris TB (2001) Race and sex effects on the association between muscle strength, soft tissue, and bone mineral density in healthy elders: the Health, Aging, and Body Composition Study. J Bone Miner Res 16(7):1343-1352

20. Verschueren S, Gielen E, O'Neill TW, Pye SR, Adams JE, Ward KA, Wu FC, Szulc P, Laurent M, Claessens F, Vanderschueren D, Boonen S (2013) Sarcopenia and its relationship with bone mineral density in middle-aged and elderly European men. Osteoporos Int 24(1):87-98

21. Kärkkäinen M, Rikkonen T, Kröger H, Sirola J, Tuppurainen M, Salovaara K, Arokoski J, Jurvelin J, Honkanen R, Alhava E (2009) Physical tests for patient selection for bone mineral density measurements in postmenopausal women. Bone 44(4):660-665 
22. Lindsey C, Brownbill RA, Bohannon RA, Ilich JZ (2005) Association of physical performance measures with bone mineral density in postmenopausal women. Arch Phys Med Rehabil 86(6): 1102-1107

23. Taaffe DR, Simonsick EM, Visser M, Volpato S, Nevitt MC, Cauley JA, Tylavsky FA, Harris TB (2003) Lower extremity physical performance and hip bone mineral density in elderly black and white men and women: cross-sectional associations in the Health ABC Study. J Gerontol Ser A Biol Med Sci 58(10):M934-M942

24. Søgaard AJ, Magnus JH, Bjørnerem Å, Holvik K, Ranhoff AH, Emaus N, Meyer HE, Strand BH (2020) Grip strength in men and women aged 50-79 years is associated with non-vertebral osteoporotic fracture during 15 years follow-up: The Tromsø Study 19941995. Osteoporos Int 31:131-140. https://doi.org/10.1007/s00198019-05191-4

25. Lundin H, Sääf M, Strender LE, Nyren S, Johansson SE, Salminen $\mathrm{H}$ (2014) One-leg standing time and hip-fracture prediction. Osteoporos Int 25(4):1305-1311

26. Kärkkäinen M, Rikkonen T, Kröger H, Sirola J, Tuppurainen M, Salovaara K, Arokoski J, Jurvelin J, Honkanen R, Alhava E (2008) Association between functional capacity tests and fractures: an eight-year prospective population-based cohort study. Osteoporos Int 19(8): $1203-1210$

27. Lim Y, Kim K, Ko SH, Cho K, Jang EH, Lee SH, Lim DJ, Baek $\mathrm{KH}$, Ha HS, Park MS, Yim HW, Lee WC, Yoon KH, Son HY, Oh KW, Kang MI (2016) Gender-and age-group-specific associations between physical performance and bone mineral density, falls, and osteoporotic fractures in Koreans: the Chungju Metabolic Disease Cohort study. J Bone Miner Metab 34(3):336-346

28. Veronese N, Bolzetta F, Toffanello ED, Zambon S, de Rui M, Perissinotto E, Coin A, Corti MC, Baggio G, Crepaldi G, Sergi G, Manzato E (2014) Association between Short Physical Performance Battery and falls in older people: the Progetto Veneto Anziani study. Rejuvenation Res 17(3):276-284

29. Barry E, Galvin R, Keogh C, Horgan F, Fahey T (2014) Is the Timed Up and Go test a useful predictor of risk of falls in community dwelling older adults: a systematic review and meta-analysis. BMC Geriatr 14(1):14

30. Jacobsen BK et al (2011) Cohort profile: the Tromsø study. Int J Epidemiol 41(4):961-967

31. Forsén L, Berntsen GK, Meyer HE, Tell GS, Fønnebø V, NOREPOS Core Research Group (2008) Differences in precision in bone mineral density measured by SXA and DXA: the NOREPOS study. Eur J Epidemiol 23(9):615-624
32. Omsland TK, Emaus N, Gjesdal CG, Falch JA, Tell GS, Forsen L, Berntsen GKR, Meyer HE (2008) In vivo and in vitro comparison of densitometers in the NOREPOS study. J Clin Densitom 11(2): 276-282

33. Roberts HC, Denison HJ, Martin HJ, Patel HP, Syddall H, Cooper C, Sayer AA (2011) A review of the measurement of grip strength in clinical and epidemiological studies: towards a standardised approach. Age Ageing 40(4):423-429

34. Podsiadlo D, Richardson S (1991) The Timed "Up \& Go": a test of basic functional mobility for frail elderly persons. J Am Geriatr Soc 39(2):142-148

35. Bergland A, Strand BH (2019) Norwegian reference values for the Short Physical Performance Battery (SPPB): the Tromsø study. BMC Geriatr 19(1):216

36. Bergh S, Lyshol H, Selbæk G, Strand BH, Taraldsen K, Thingstad P (2013) Short Physical Performance Battery (SPPB) Norwegian version. Legeforeningen. Available at: https://www. $\mathrm{leg}$ e for e n i nge n. no/ conte n t a s s e t s/ 870420284b7d4cb98100191ff93e7983/sppb.pdf. Accessed 16 April 2021

37. Kuh D, Bassey EJ, Butterworth S, Hardy R, Wadsworth MEJ, and the Musculoskeletal Study Team (2005) Grip strength, postural control, and functional leg power in a representative cohort of British men and women: associations with physical activity, health status, and socioeconomic conditions. J Gerontol Ser A Biol Med Sci 60(2):224-231

38. Sagelv EH, Hopstock LA, Johansson J, Hansen BH, Brage S, Horsch A, Ekelund U, Morseth B (2020) Criterion validity of two physical activity and one sedentary time questionnaire against accelerometry in a large cohort of adults and older adults. BMJ Open Sport Exerc Med 6(1):e000661

39. Grimby G, Börjesson M, Jonsdottir IH, Schnohr P, Thelle DS, Saltin B (2015) The "Saltin-Grimby Physical Activity Level Scale" and its application to health research. Scand J Med Sci Sports 25:119-125

40. Denk K, Lennon S, Gordon S, Jaarsma RL (2018) The association between decreased hand grip strength and hip fracture in older people: A systematic review. Exp Gerontol 111:1-9

Publisher's note Springer Nature remains neutral with regard to jurisdictional claims in published maps and institutional affiliations. 